\title{
沉浸式可视化综述
}

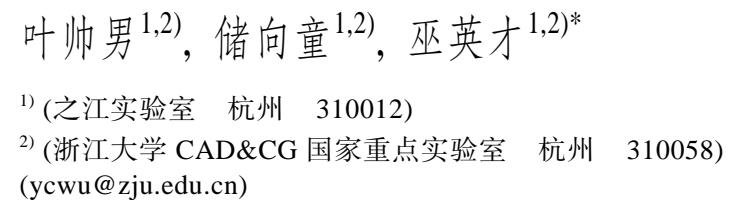

摘 要: 沉浸式可视化是一个新兴的研究领域, 旨在通过沉浸式交互界面所提供的沉浸感与参与感充分运用人们的 感知与操作能力, 为数据分析推理与决策提供支持. 以最新的沉浸式可视化研究为基础, 首先从沉浸式感知的评估 与运用出发, 介绍视觉感知以及非视觉辅助感知在信息可视化中的应用方式与评估结果; 然后对于可视化中的交互 设计, 分别从触摸、手势、注视与移动导航 4 种类别概述最新的交互技术; 再针对可视化的创作工具, 从桌面端、移 动端以及多端融合 3 个方面进行创作工具的概述; 最后对沉浸式可视化的产业趋势、应用以及挑战进行总结.

关键词: 沉浸式可视化; 人机交互; 自然用户界面; 创作工具; 虚拟现实; 增强现实; 可视分析 中图法分类号: TP391.41 DOI: 10.3724/SP.J.1089.2021.18809

\section{A Survey on Immersive Visualization}

\author{
Ye Shuainan $^{1,2)}$, Chu Xiangtong ${ }^{1,2)}$, and Wu Yingcai ${ }^{1,2)^{*}}$ \\ 1) (Zhejiang Laboratory, Hangzhou 310012) \\ 2) (State Key Laboratory of CAD\&CG, Zhejiang University, Hangzhou 310058)
}

\begin{abstract}
Immersive visualization is an emerging research field. It aims to make full use of human perception and operation capabilities through the immersion and engagement offered by the immersive interface to support data analysis, reasoning, and decision-making. The latest research on immersive visualization is reviewed. Firstly, the design and evaluation of the visual and non-visual perception in information visualization are introduced. Secondly, the newest interaction methods from the aspects of touch, gesture, gaze, and navigation are summarized. Afterward, the visualization authoring toolkit in terms of desktop, mobile, and mix terminals is overviewed. Finally, the industry trends, applications, and challenges of immersive visualization are discussed.
\end{abstract}

Key words: immersive visualization; human-computer interaction; natural user interface; authoring tool; virtual reality; augmented reality; visual analytics

可视化是一种数据分析与挖掘的重要科学方 法, 在生物应用 ${ }^{[1]}$ 、城市规划 ${ }^{[2] 、}$ 物理流体 ${ }^{[3]}$ 和体 育人文 ${ }^{[4]}$ 等关键领域发挥着巨大的作用. 它通过基 于计算机的可视化系统为人们提供数据可视化表
达, 旨在让人们参与到数据分析的过程中, 增强人 们理解与操作数据的能力 ${ }^{[5]}$. 随着大数据时代的到 来, 可视化赋予人们从大量数据中快速发现关键 信息, 从而提升各种复杂任务完成效率, 成为大数

收稿日期：2020-11-24; 修回日期：2021-01-04. 基金项目：国家自然科学基金(62072400, 61761136020, U1609217); 浙江省自然 科学基金(LR18F020001). 叶帅男(1993一), 男, 博士研究生, 主要研究方向为沉浸式可视化、可视分析、人机交互; 储向童(1998一), 女, 博士研究生, 主要研究方向为可视分析、计算体育; 巫英才(1983一), 男, 博士, 研究员, 论文通讯作者, 主要研究方向为可视分 析、信息可视化、人机交互. 
据处理的关键技术 ${ }^{[6]}$.

为了让人们融人分析的过程, 可视化系统需 要提供包括数据的可视化表达, 使人们能够通过 视觉等感知快速获取数据中有价值的信息以及交 互方式, 使人们能够将专业领域知识融人分析过 程，提升决策的有效性. 然而, 无论可视化表达或 交互方式的提供都需要交互界面的支持. 依据目 前普遍接受的定义, 可视分析是一门通过交互界 面促进的分析推理科学 ${ }^{[7]}$. 传统的交互界面设备, 无论加载何种可视化系统, 皆以二维显示屏和鼠 标键盘构成为主. 为此, 可视化设计无论在数据映 射通道或是交互方式的设计上都以平面为基础.

近年来，沉浸式技术的发展为可视分析提供 了一类新的交互界面设备, 如 Oculus Rift, HTC VIVE 和 Microsoft HoloLens 等. 沉浸式技术所提 供的交互界面具有沉浸感与参与感 2 种核心特性 ${ }^{[8]}$. 其中，沉浸感包括用户在交互空间中产生的主观 临场体验，以及呈现这种空间所需的技术的客观 特征 ${ }^{[9-10]}$; 参与感则描述用户利用这种交互界面进 行数据分析任务的专注程度 ${ }^{[11]}$. 随着沉浸式技术 的进步, 如更加广阔的视野和具象化的交互设计, 沉浸式技术逐步向着为用户提供更加接近真实物 理环境的虚拟交互空间迈进. 最新的研究通过挖 掘诸如视觉深度信息 ${ }^{[12-13]}$ 、触觉反馈 ${ }^{[14]}$ 进一步利 用用户的三维控制感与真实感，进而提升用户在 处理各种任务中的效率. 相比于传统桌面端的交 互界面，沉浸式交互界面能够利用人们的立体视 觉展现数据, 将数据表达空间从二维平面转向用 户周围的三维空间; 提供可移动性, 从而让用户的 物理工作环境不再限于固定的办公桌面; 提供如 手势等自然的交互方式，使交互更加直观；以及提 供触觉、嗅觉等交互通道, 让用户利用多通道自然 而并行地与数据交互. 越来越多的研究结果表明, 真实感的三维交互界面在数据的探索、理解与分析 中存在着巨大的潜力.

沉浸式技术有着较长的历史, 最早的沉浸式 设备可追溯至 1962 年的 Sensorama. 然而, 在可视 化领域的顶级会议中, 首次出现沉浸式的讨论是 IEEE VIS 2014 年的研讨会“台式机的死亡：构想 脱离台式机的可视化”. 随后, 有关沉浸式可视化 或沉浸式分析的研讨会在各种会议中陆续出现, 如 IEEE BDVA 与 IEEE VIS.

沉浸式可视化的研究包括设计与评估新颖的 可视化展现与交互方式，以及创作这些可视化的
工具设计与开发. 一方面, 这些设计能够利用沉浸 式的感知特性提升用户理解、探索与分析数据的效 率; 另一方面, 设计工具不仅能够让用户便捷地实 现沉浸式可视化, 还能降低其设计与使用的门槛, 从而推进可视化的普及, 让可视化叙事融人工作、 传播和日常生活中. 基于这些优势, 研究者根据人 们的感知特征, 提出了丰富的可视化设计; 从人们 日常的交互动作出发, 探索自然且高效的交互技 术; 以新场景、新用户的特点, 研制了便捷的开发 工具. 这些成果共同提升了人们处理复杂任务的 能力. 与此同时, 如何进一步理解多通道感知对于 可视化分析的作用, 并以此解决不同场景下的新 需求, 将沉浸式可视化融人更为广阔的应用领域 中, 则尚待研究与挖掘.

\section{1 沉浸式感知与可视化}

近年来，消费级沉浸式设备的发展为可视化 研究带来了许多机遇. 沉浸式设备将用户置于三 维空间之中. 一方面, 这不仅释放了用户的立体视 觉, 也释放了触觉、本体感知等能力, 从而让可视 化编码与交互不再局限于二维视觉通道与视觉反 馈; 另一方面, 也极大地拓展了设计空间, 如基于 球面的布局 ${ }^{[15]}$ 以及三维物体与二维可视化融合的 嵌人式视图可视化 ${ }^{[16]}$. 这些沉浸式空间所带来的 感知与改变, 让研究者开始发掘沉浸式交互空间 在可视化与可视分析中的潜力.

本节从可视化所利用的感知类别进行分类, 分别阐述视觉感知以及非视觉感知在三维沉浸空 间中进行信息可视化的最新设计与评估成果.

\section{1 三维视觉感知}

人们对三维的视觉感知来自各种深度提示. 这 些提示主要包括双目视差、遮挡和相对大小等 ${ }^{[17]}$, 其对可视化有利弊两方面的影响. 一方面, 三维中 的视角倾斜会使二维平面图形(如文字和图符)产 生形变, 用户难以识别 ${ }^{[5]}$; 另一方面, 人们能够轻 易地识别三维物体的形态, 因此科学可视化中常 以此对三维形态数据进行分析. 由于三维空间中 存在广大的设计空间, 因此, 研究者逐渐探索出许 多能够扬长避短的可视化设计.

点是可视化中的重要标记, 其通常与位置视 觉通道一起表示 1 3 个属性, 如二维散点图. 当存 在大量数据点时, 聚簇的寻找与识别成为数据探 索的重要任务. Kraus 等 ${ }^{[18]}$ 发现, 与二维屏幕相比, 
用户在虚拟现实(virtual reality, VR)环境下对三维 散点图的聚类不仅具有更高的识别率，也更易于 记忆. 此外, 为了在众多的点或物体中突出重要的 个体, 可视化系统需要提供额外的视觉提示, 从而 让视觉标记吸引用户注意. 然而, 传统的方式, 如 改变物体的颜色或闪㷧等高亮方法，可能会占用 原有的视觉通道. 利用三维空间提供的深度信息, Alper 等 ${ }^{[13]}$ 提出将待高亮标记的位置朝视野方向靠 近的方法, 用户利用立体视觉感知近处的标记达 到高亮数据的效果. 然而, 位置的移动会占据重要 的位置视觉通道. 如图 1 所示, 最近, Deadeye $\mathrm{e}^{[19-20]}$ 巧妙地利用分裂呈现方法, 将待高亮的标记只显 示于其中一只眼中, 以对双眼形成不同的刺激达 到凸显的效果. 该方法不仅不会占据视觉通道，同 时高亮的识别效果不受原本视觉符号编码通道的 影响.

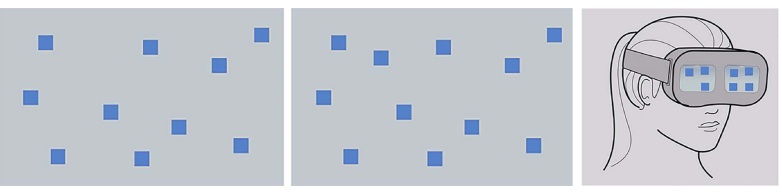

a. 双眼看到各自不同的内容

b. VR 示意图

图 1 分裂呈现方法示意图

时空轨迹数据有着广泛的应用, 如起论点流 图蕴含着城市中人口流动的规律，反映了交通结 构的合理性. 然而，传统的流图将同一区域不同时 间的流动都投影至一片区域中，造成不同时刻流 动情况相互覆盖. 一种利用三维空间的改进方法 是使用时空立方体，其中水平平面对应事件发生 的位置空间，而用高度表示事发时间. 如图 2a 所 示, GeoGate ${ }^{[21]}$ 将时空立方体置于桌面地图上方的 增强现实(augmented reality, AR)空间中，并在其周 围辅以立方体中的轨迹投影，从而降低用户对复 杂轨迹观察的难度. 如图 2b 所示, Wagner Filho 等 ${ }^{[22]}$ 在 VR 中将轨迹与桌面的地图联系在一起, 并设计 相应的手势操作，使用户能够便捷地观察轨迹在 时序上的变化, 从而缓解人们理解与探索时空立 方体中三维轨迹的困难. Yang 等 ${ }^{[23]}$ 利用第 3 维度寻 找起论点流图的合理设计，通过对比不同流图可 视化以及不同的参考空间，发现利用高度编码流 量大小属性, 并且如图 2c 所示使用虚拟地球仪代 替传统的平面地图作为对应的参考空间，在流量 大小对比上具有更高的效率，从而验证了利用第 3 维度的合理编码降低视觉混乱的可行性.

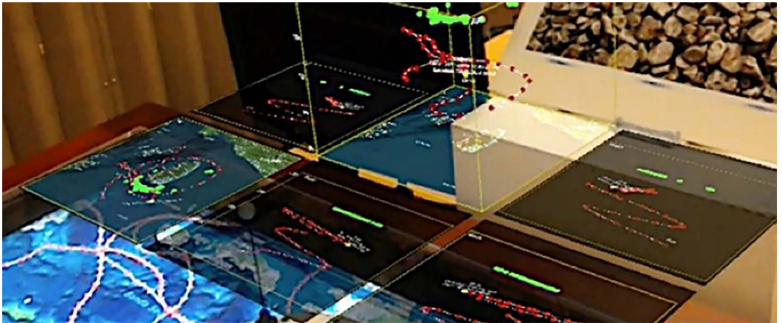

a. GeoGate AR 时空立方体

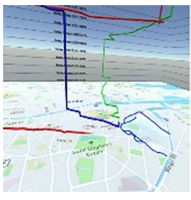

抓

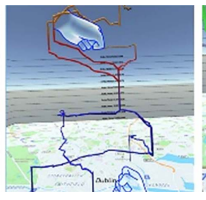

拉

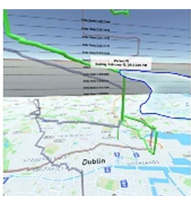

轻击

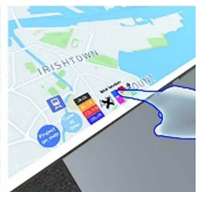

点击虚拟桌面 b. 引人三维轨迹的桌面地图

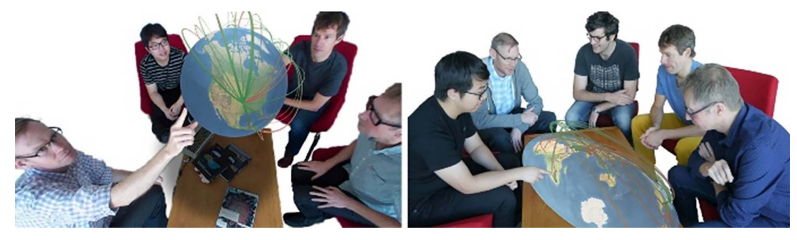

地球仪与起论点流图

地图与起论点流图

c. 三维起论点流图设计

图 23 种三维轨迹可视化设计

点与边构成的图在信息可视化中有着重要的 应用，如展现个体之间的联系以及社群中的交往 结构. 传统的图布局受到二维空间的局限, 当点与 边较多时容易存在较大的视觉混乱, 影响用户对 图数据的理解. Kwon 等 ${ }^{[12]}$ 提出了利用三维空间中 球面进行顶点的布局, 并使用边绑定的方式将边 置于球面外侧，让用户从球中心进行观察，如图 3 所示. 实验结果表明, 基于球面的顶点布局不仅避 免了边对点的遮挡, 立体视觉以及更大的视野范 围也有效地减轻了边之间的视觉混乱, 提升了用 户对于大数量级图中复杂路径寻找任务的完成效 率. 然而, 随着数据属性的增加, 边不仅要表示点 的链接, 还需要表示诸如流量和方向等属性. 为了
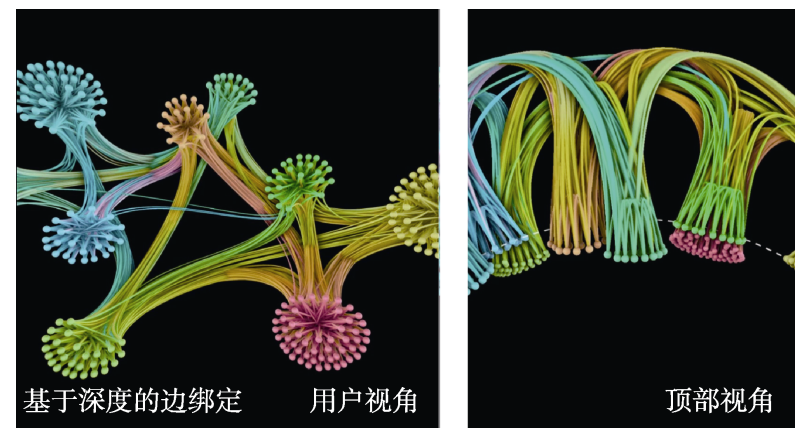

图 3 基于球面的顶点布局 
探寻边属性的可视化方法, Büschel 等 ${ }^{[24]}$ 利用边的 曲率(直线或曲线)、粗细以及图符(如虚线类型)等 6 类可视变量进行边属性的编码, 发现这些编码方 式都可用于有向图或无向图中的路径搜寻任务. 然而, 边的可视变量种类繁多, 如何寻找适合于大 尺度图的边属性可视化方法依然有待探索.

除此之外, 研究者也尝试对其他传统的可视 化图表进行三维的扩展, 评估用户使用的效率以 及解决现有的难点. 针对二维热力图在对比时使 用并列放置的方法难以对特定区域进行数值比较 的问题, Kraus 等 ${ }^{[25]}$ 设计了三维热力图的对比系统; 利用颜色和高度的双重编码生成三维热力图, 同 时允许用户调整热力图的重叠程度交互地完成对 比, 如图 4a 所示. 实验结果表明, 叠加三维热力图 比传统热力图更有利于支持特定区域值比对、相似 性区域搜寻等需要局部区域精确比较的任务. Ivanov 等 ${ }^{[26]}$ 将二维人物图标扩展至三维化身, 让用户 处于化身所在的三维空间中, 并赋予三维化身尺 度变换的功能, 以实现全局与细节的观察，从而展 现与人物群体有关的数据故事. 用户实验表明, 利 用化身的拟人特性, 能够加深用户对数据故事的 情感理解. 针对三维物理数据与二维抽象数据融 合显示的问题, Chen 等 ${ }^{[16]}$ 提出了图 4b 所示包括链 接视图、嵌人视图和混合视图 3 种布局方法. 同时, 从二维抽象数据与三维物理数据之间的几何形态 以及空间相对位置是否接近这 2 个维度, 讨论了每 种视图的适用情况, 为可视化设计者提供了各视 图的使用指南.

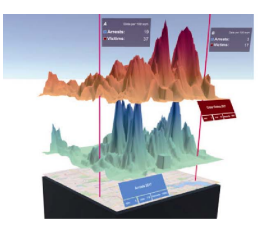

较少重叠

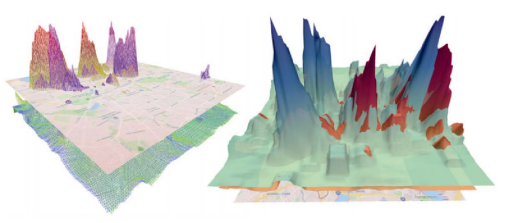

较多重叠

a. 叠加三维热力图的对比

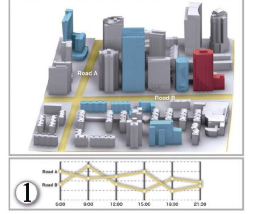

链接视图

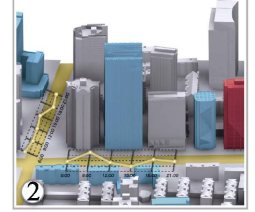

嵌人视图

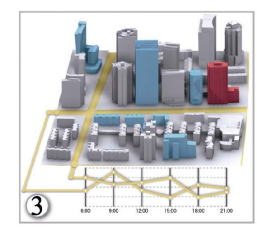

混合视图 b. 整合物理数据与抽象数据的布局方案

图 4 传统图表的三维扩展

\section{2 非视觉感知的辅助}

除了视觉感知外，人们对于三维空间的感知 还包括触觉、嗅觉以及本体感知等, 这些知觉都可
被利用或影响数据的编码与表达. 然而, 与视觉相 比, 这些感知各自传递的信息十分有限, 因此往往 与视觉配合使用, 作为辅助视觉的信息传达通道, 并提升用户的沉浸感与参与感.

通过振动幅度大小的等级, 人们能够使用触 觉感知一维离散数据信息. Prouzeau 等 ${ }^{[14]}{ }^{1}$ 针对三维 海量散点图中寻找高低密度区域的困难, 提出利 用手柄设备提供的震动功能产生对散点区域密度 的触觉反馈, 从而提升用户对聚簇识别与对比的 效率. 如图 5 所示, 该方法将散点图中的离散点转 换为连续的密度体, 并将手柄所在区域的密度大 小映射为手柄震动的强度高低, 使用户能够通过 握住手柄“触摸”散点空间, 完成如图 5 所示 $C_{1}, C_{2}$ 和 $C_{3}$ 聚簇所在区域及其密度相对高低的判断.

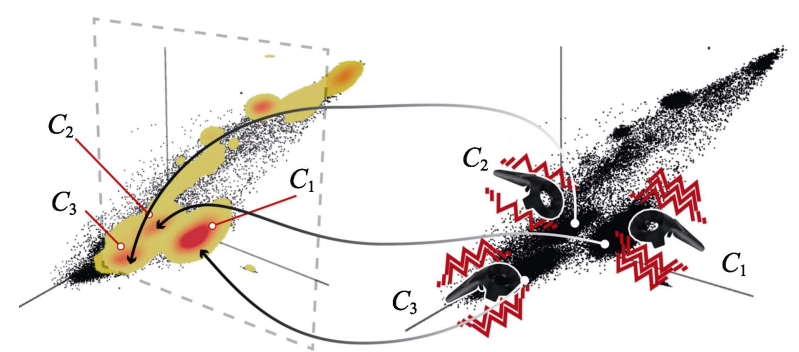

图 5 散点图中区域密度与手柄震动强度的映射

嗅觉是另一个基本的感知通道, 其具有易于 记忆和识别的优势. 基于此, viScent ${ }^{[27]}$ 使用用户的 嗅觉感知传递数据信息, 作为视觉信息的自然补 充, 以增强用户对信息的理解与记忆. 该方法类比 于可视化中的可视化标记与视觉通道, 提出了由 嗅觉标记(如各种气味) 以及嗅觉通道(如强度、空气 流动速率与温度等)构成的数据映射设计空间, 进 而将数据映射为对应的气味属性, 辅助视觉提升 用户对数据的理解.

听觉感知通道能够为用户提供丰富的信息, 如以语音传递的语义信息 ${ }^{[28]}$ 和以立体声表达的空 间几何信息 ${ }^{[29]}$. 然而, 在沉浸式环境中, 将听觉感 知通道用于数据表达的工作较为欠缺, 仍待研究 者的探索.

除此之外, Batch 等 ${ }^{[30]}$ 分析了用户如何利用三 维空间进行多维经济数据的探索以及结果的展示. 在实验中, 用户通过沉浸式工具 $I m A x e s^{[31]}$ 在三维 工作空间中创造并放置多个可视化视图, 如生成 简单常见的平行坐标图以及较为复杂的三维散点 图. 用户同时能够将这些视图按用户喜好摆放至 身体周围, 以方便后续整理. 此过程不仅需要视觉 
感知, 还需要运用用户的三维感知(如本体感知、 空间记忆). 该研究发现, 合理的系统设计能够部 分抵消三维可视化的缺陷. 例如, 文字的可读性在 实验中并未受到明显影响; 同时用户能够按照探 索的需要排列虚拟的视图支持回忆与聚类, 并经 常使用更加复杂的可视化视图讲解分析结果.

\section{2 沉浸式交互的设计}

交互能够为各类型分析任务(如数据选择、参 数调整、视角导航等)提供基本的支持, 在沉浸式 可视化与可视分析中至关重要. 现有的研究重点 集中于探索更加自然的交互方式，以提升用户在 三维空间中的操作效率. 具体而言, 这类交互界面 试图通过识别用户的手势、手臂和身体移动等动作 所表达出的物理隐喻完成对应的任务，让用户与 数据的交互变得更为自然 ${ }^{[32]}$. 按照人们日常与物 体的交互方式，这类方法可大致分为基于接触，即 通过触碰标记以传达数据操作; 基于手势, 即通过 手部或手柄的动作以表达交互内容; 基于注视，即 用眼部的注视产生交互; 以及基于移动导航，即通 过身体移动完成交互操作. 因此, 本节主要从这 4 种方法出发，对沉浸式交互技术进行综述.

\section{1 基于接触的交互}

在基于接触的交互方法中，用户通过手部或 手持传感器与可视化标记直接触碰, 从而传递与 该标记数据的交互表达. 在沉浸式设备兴起之前, 这类交互主要用于触屏式设备中, 如平板电脑和 手机终端; 数据直接显示于屏幕, 用户通过对触屏 的点击、滑动完成对数据的选择等操作 ${ }^{[33]}$. 随着 $\mathrm{AR}$ 在手机和平板设备中的使用, 此类操作具有操 作精度高的特征，因此许多工具利用触屏完成布 局等与位置操作有关的任务. 例如, Pronto ${ }^{[34]}$ 使用 平板设备精准绘制出如图 6a 所示蓝色动画移动曲 线; 而 MARVis ${ }^{[35]}$ 如图 $6 \mathrm{~b}$ 所示利用点击、拖动将 图标在手机所处的平面上进行布局.

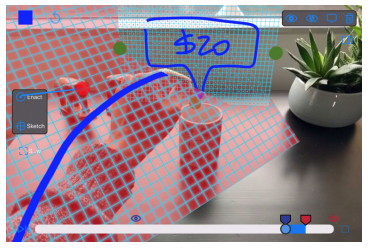

a. Pronto 曲线绘制

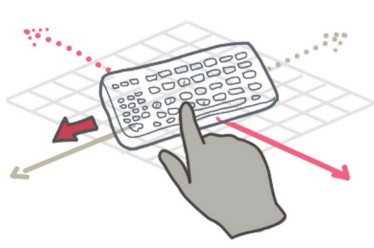

b. MARVisT 平面布局物体
图 6 触屏式设备的接触交互设计
除了基于屏幕的触摸外，基于接触的交互在 头戴式沉浸设备中也十分常见. 例如, 在 VR 环境 中，设备通过手部跟踪捕获用户勾勒的脑神经路 径 ${ }^{[36]}$. ShuttleSpace ${ }^{[4]}$ 根据现实中羽毛球运动员的 击球动作, 设计了 VR 环境中基于击球隐喻的羽毛 球轨迹交互方法, 让教练能够从大量轨迹中快速 而准确地完成轨迹篮选操作. 在选择时, 教练手握 控制器于击球处, 并如运动员一样朝着击球点完 成一次挥拍击球动作. 随后, 系统通过获取与击球 点触碰时的速度与位置, 从轨迹中选取与之最接 近的轨迹, 如图 7a 所示. 基于现实动作隐喻的交 互不仅操作直观，让教练等专业人员易于上手，而 且富有表现力, 因为教练足以选择他们想要的轨 迹. 然而, 在 AR 环境中, 虚拟的物体以覆盖的方 式置于真实物体之前, 这导致判断手与被交互物 体之间的相对位置造成困难. 为此, Wacker 等 ${ }^{[37}$ 将 虚拟物体与手部的距离映射为颜色的色调并显示 于虚拟物体上，形成了由虚拟物体构成的热力图， 如图 7b 所示. 用户通过识别颜色判断手与物体的 相对位置，提高选择的准确性.

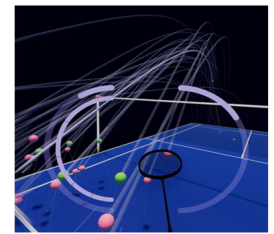

用户位于击球处

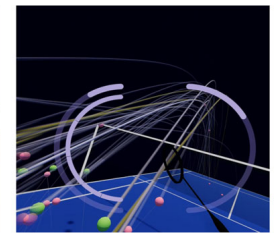

挥拍篮选黄色轨迹

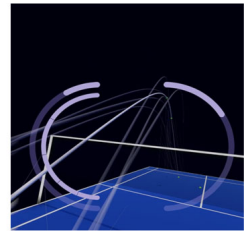

保留选取轨迹 a. 基于挥拍隐喻的羽毛球轨迹交互

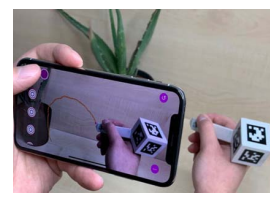

$\mathrm{AR}$ 设备

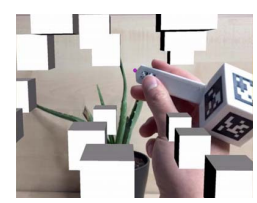

虚拟物体覆盖手笔

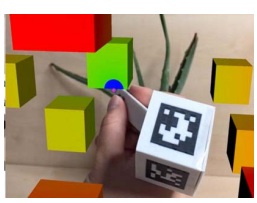

颜色视觉提示 b. 利用热力图颜色辅助相对位置判断

图 7 基于虚拟物体接触的交互

与数据交互后产生的触觉反馈对于交互中的 沉浸式体验至关重要, 它能够让用户确信交互动 作已产生效果, 进而提升交互的直观性与沉浸感. 然而，沉浸式空间中的可视化标记大多为虚拟物 体, 天然缺乏触觉反馈. 为此, Prouzeau 等 ${ }^{[14]}$ 利用 手柄设备所提供的震动功能, 将数据点密度编码 为震动强弱, 让用户以视觉与触觉感知并行的方 式完成散点图的探索.

除了震动的反馈，研究者还探索利用真实物 体与虚拟物体相结合的方式提升虚拟物体的真实 
感. 具体而言, 该方法将现实中的可接触物体作为 虚拟标记在物理世界中的参照物. 用户通过触碰 真实物体来增加交互的精确性. 例如, 研究者利用 虚拟桌面隐喻 ${ }^{[22,38-39]}$, 将沉浸空间中的地图或数 据投影等二维可视化所在的平面映射为真实桌面, 从而让用户点击物理桌面操作对应数据, 分别如 图 2b 和图 8a 所示. 此外, 将有形用户界面运用于 沉浸式交互的方法开始逐渐兴起. Cordeil 等 ${ }^{[40]}$ 通 过 3 个滑块轴将数据坐标轴实物化, 如图 8b 所示. VR 设备将三维可视化显示于滑块轴所包围的空间 内, 用户直接操纵实物坐标轴上的驱动滑块精准 地选择轴空间内的数据.

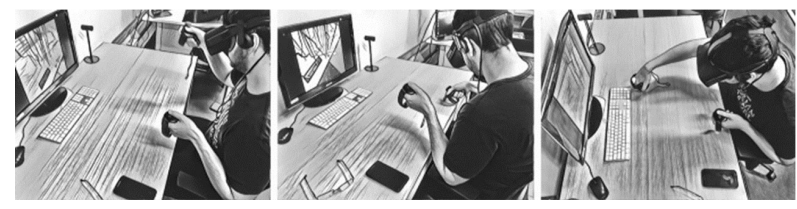

与空中虚拟物体交互

与桌面上的二维图表交互

a. 虚拟桌面使用示意图

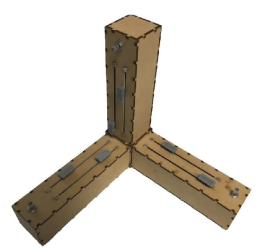

实物化坐标轴

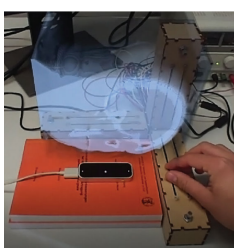

三维体切片

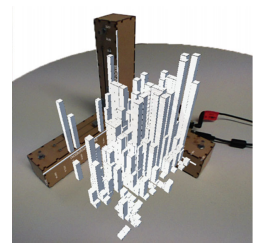

柱状图可视化 b. 有形用户界面

图 8 具有触觉反馈的交互

\section{2 基于手势的交互}

手势是人们日常交互的手段之一. 例如，通过 指向目标和手的平移，人们可以自然地表达选择、 移动图片等语义信息. 在沉浸式环境中, 交互界面 主要使用可跟踪设备(如手柄)或对手指动作的捕 捉, 从而识别手部所做出的动作, 让用户从远距离 完成数据的操控 ${ }^{[32]}$. 这使得交互空间不再限于手 臂范围之内 ${ }^{[41]}$, 也降低了用户选择远距离数据所 需的导航距离.

基于光线投射的目标指向是一种常见的数据 选择技术，其通过手柄等装置的朝向射出光线，选 择与光线相交的最近物体. 然而, 远距离物体的选 择存在诸多困难, 包括较小的目标物体、精准的手 部姿态控制、物体的相互遮挡以及选择物体所需的 位置难以导航等. 为了增加选择点物体的精确度, RayCursor ${ }^{[42]}$ 在传统光线投射指向技术中增加了沿 投射光线方向的控制光标，并将离光标最近的物 体视为选中，以有效地避免被遮挡物体的选择，如
图 9a 所示. 该方法同时使用滤波缓解手部的抖动, 提升远距离物体选择的效率. 此外, FiberClay ${ }^{[43]}$ 通 过双手操控从手柄投射的光线逐步篎选密集轨迹 数据. 用户利用左右手 2 束光线的配合与空中轨迹 或地面起论点相交完成轨迹的过滤, 同时展现轨 迹的多个二维投影, 从而在各维度上逐步篮选出 目标轨迹.

除了基于指向隐喻的光线投射技术，其他隐 喻(如抓取、拖动等)在沉浸式环境中的应用都有相 关研究. Satriadi 等 ${ }^{[44]}$ 针对 AR 中空中手势对于地图 导航所存在的操作精度不足、易于疲劳的问题，设 计了抓取和拖动结合的手势，同时完成地图平移 与缩放操作. 此交互方式将手部周围的空间划分 为多个区域, 用户只需要将手偏移至指定区域, 使 用抓取动作即可完成对应操作. 图 9b 展示了一个 缩放操作的例子：用户在空中抓住并坚直离开起 始区域触发缩放地图操作, 随后水平平移完成地 图缩放的同时平移地图. 除此之外, Yang 等 ${ }^{[45]}$ 将双 手之间的距离映射至三维散点图展示的尺度, 通 过双手的合拢与展开实现散点图的缩放操作; Tilt $\mathrm{Map}^{[46]}$ 利用手柄的倾斜角度变化, 实现如图 $9 \mathrm{c}$ 所 示地图图表在分级统计图、棱镜图与二维柱状图之 间的视图转变, 使用户能够快速地选择不同视图, 同时观看数据在各视图间的变化, 减轻用户在视 图切换时的理解难度.

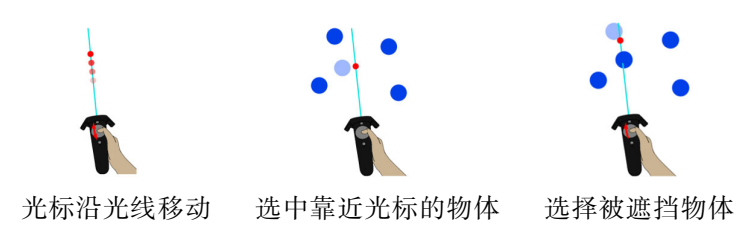
a. 利用光标选择物体
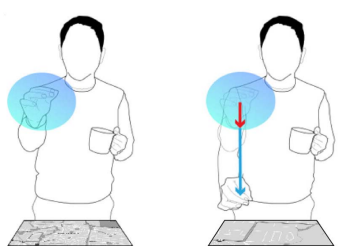

缩放平移地图操作

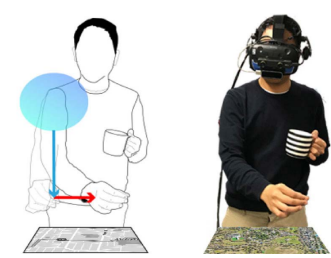

用户穿戴设备 b. 抓取、拖动结合的地图交互
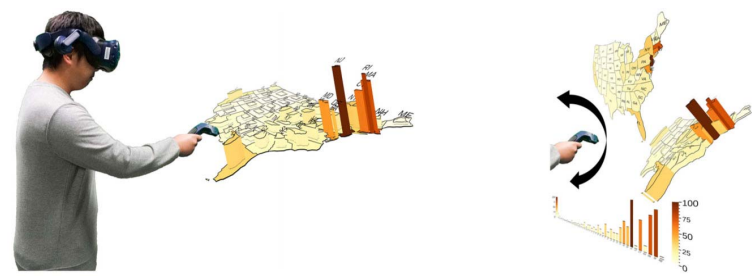

c. 用户倾斜手柄实现多视图观察

图 9 基于手势的交互设计 


\section{3 基于注视的交互}

基于注视的交互主要是基于光线投射隐喻的 目标指向技术，常用于快速选取物体以及代替手 部操作，让其同步完成其他任务. 通过眼动跟踪, 交互界面能够获得用户所表达的诸如导航方向和 物体轮廓等语义信息. Outline Pursuits ${ }^{[47]}$ 利用物体 遮挡时露出的轮廓，如图 10 所示在候选目标上增 加沿轮廓移动的圆点. 系统通过让用户注视圆点 并同时捕获眼球的移动，将被注视圆点所在的物 体识别为用户的选择结果，降低对被部分遮挡物 体的选择难度. 此外, 通过识别用户观看的场景以 及之前观看的顺序, Kwok 等 ${ }^{[48]}$ 为用户自动推荐合 适的解说条目，自适应地为用户可视化其正在关 注的内容.

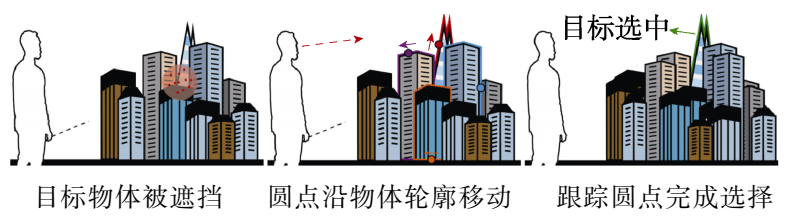

图 10 Outline Pursuits 利用眼球跟踪提示完成选择

眼球的移动不仅能够主动表达交互，还能够 显示用户的心理状态，从而被动地触发交互. 例 如, Alghofaili 等 ${ }^{[49]}$ 通过识别眼球的移动规律, 判 别用户在虚拟环境中是否迷失，进而自适应地显 示辅助标志. 此方法首先采集用户的注视序列以 及请求提示的标记训练长短期记忆网络 (long short-term memory, LSTM)模型, 并以此模型完成 用户迷失状态的检测.

\section{4 基于移动导航的交互}

基于移动导航的交互主要用于改变用户在三 维空间中的位置, 从而寻找最佳的视角观察数据. 由于穿戴在用户身上的设备具有实时定位功能, 因此最直接的视角导航方式即是用户在物理空间 中的移动，如身体的走动以及头戴设备下头部的 转动. 然而, 虚拟空间与物理空间的不匹配, 如虚 拟空间大于用户的可移动物理范围, 会导致无法 获得最佳的观察视角, 阻碍对数据的探索能力. 为 此, 研究者提出物理移动与虚拟移动的映射方法. 例如, Abtahi 等 ${ }^{[50]}$ 设计了 3 种真实与虚拟移动速度 的映射层次，解决虚拟空间大于真实空间的导航 问题. 其中, 每种层次有各自从物理速度到虚拟速 度的缩放程度，从而让用户获得在虚拟环境中比 物理空间而言更远的移动, 降低物理空间大小的 限制。

除了物理空间的移动获取视角导航，交互设
备还支持虚拟移动技术. 一种常见的方法是基于 传送的导航, 其中用户首先指定希望移动的位置 点, 随后虚拟位置立刻完成切换. 例如, Funk 等 ${ }^{[1]}$ 通过增加传送后朝向的控制增加了传送的精度; Yang 等 ${ }^{[45]}$ 在三维缩略图中增加用户在虚拟空间的 实时位置以及朝向的图标，并支持用户拖拽图标 或直接点击缩略图中的位置完成传送. 除此之外, 研究者还提出飞行隐喻的导航. Drogemuller 等 ${ }^{[52]}$ 介绍了包括单手与双手的飞行模式，通过手柄的 朝向(单手)或手柄之间的向量构成飞行的方向(双 手), 让用户在三维图中实现导航探索.

\section{3 沉浸式可视化创作工具}

沉浸式技术的快速发展丰富了可视化的作品, 也衍生出大量可视化创作工具. 就现有的可视化 系统而言, 这些可视化作品一般由技术人员运用 专业工具(如 AR Core, D3 等)编写而成. 然而, 这 些工具不仅需要使用者具备成熟的编程技巧，而 且需要花费大量的时间编程与设计. 近年来, 为了 降低可视化的设计与使用门槛, 推进可视化的普 及, 让可视化创作融人日常生活中, 研究者开始提 出许多轻量级的创作工具. 本节将根据创作所需 的设备分别介绍最新的沉浸式可视化创作工具.

\section{1 基于桌面端的工具}

基于桌面端的创作工具支持用户迭代地在桌 面端上设计开发并在终端(如手机、VR 设备)上展 示创作结果. 借助以 Unity 为首的桌面端开发引擎, 许多沉浸式可视化创作工具也以桌面端为开发平 台, 为用户提供灵活的跨平台开发解决方案以及 简洁易上手的编辑器等支持. 为了降低可视化创 作烦琐的编程负担，这类创作工具将创作接口分 为面向新手、用于简单创作的高层图形界面，以及 为具有专业知识用户提供的用于复杂创作的底层 语法界面

Sicat 等 ${ }^{[53]}$ 提出面向大众使用且支持快速构建 沉浸式可视化的工具包 DXR. 当前设计人员在创 作可视化时需要手动编写专业代码实现数据与可 视化之间的映射关系. 为了减轻编写的负担, DXR 预先声明了可视化语法, 设计人员只需依照语法 导人数据属性与可视化配置信息即可建立视觉映 射，并可使用沉浸式设备实时预览可视化效果．同 时, DXR 提供了用于快速编辑可视化相关配置的 图形界面. 除此之外, 它还支持自定义图形标记, 从而实现沉浸式可视化的个性化创作. 
IATK $^{[54]}$ 除了支持可视化创作之外，还允许设 计人员与沉浸式环境的直接交互. 类似于 DXR, IATK 是一个发布在 Unity 社区中的开源工具包, 用于协助用户快速创建沉浸式可视化的数据分析 系统; 不同的是, 它更加侧重后期在沉浸式环境中 与三维数据的交互. 借助手势识别, 设计人员能够 在已创建的可视化沉浸式环境中对数据进行手动 旋转、缩放和笁选等交互设计.

\section{2 基于终端的工具}

由于基于桌面端的创作工具的创作过程脱离 沉浸式终端设备, 设计人员无法即时查看设计结 果获取反馈, 并且需要在设备之间来回切换. 相比 之下，基于终端的创作工具允许用户直接在终端 上完成沉浸式可视化的设计与预览, 减轻了切换 的负担并降低了潜在设计错误的风险.

MARVisT $^{[35]}$ 是用于个人信息的可视化创建工 具，它允许用户使用手机或平板电脑等终端将虚 拟图符与真实物理对象绑定, 完成在 AR 中对被绑 定物理对象的虚拟图符可视化. 用户在经过视觉 映射的选择以及绑定之后, 系统能够自动依据真 实物体在视野中的大小与位置完成虚拟图符的尺 度缩放与布局. MARVisT 让数据以图符的形式直 观地展示在用户面前, 促进用户对数据的理解与 表达.

\section{3 多端融合的工具}

基于终端的可视化创作工具限于终端的功能, 往往仅支持简单的可视化设计, 同时需要设计师 在 AR 可视化的现场完成, 阻碍了远程设计的方 式. 多端融合则尝试结合桌面端与终端的优点, 实 现多人可视化创作. Prouzeau 等 ${ }^{[55]}$ 提出 AR 与 VR 设备融合的合作创作模式，设计师在远程使用 VR 设备设计，而终端用户在现场使用 AR 设备完成体 验并即时反馈设计结果. 该方法既能够即时地获 取可视化在真实使用时的效果，又无需设计师到 达现场完成设计. 但该方法需要在 VR 中模拟完整的 真实场景, 对创作的前期准备要求较高. 因此, 多端 融合的可视化设计仍有较大的设计与提升空间.

\section{4 结 语}

沉浸式交互界面为用户提供了比传统界面更 广阔的设计空间以及更丰富的感知通道, 从而为 可视化与可视分析提供了新的技术、场景与应用. 本文对于沉浸式可视化的最新进展进行综述，阐 述了沉浸式交互界面的特点, 并从可视化的设计、
交互以及创作工具方面介绍最新的领域进展，当 前的沉浸式设备还未成熟, 但其广阔的应用场景 与领域为未来沉浸式可视化的发展提出了新的机 遇与挑战

\section{1 沉浸式产业化趋势}

目前的沉浸式产业技术趋势分为 2 类. 一类是 以 CAVE $2^{[56]}$ 为代表的大型固定沉浸式可视化设备. 此类型设备由众多显示屏所构成的环形墙面显示 器构成, 具有极高的、可扩展的分辨率, 能够让分 析者清晰地观察数据的三维结构, 同时也支持二 维数据的展示. 由于造价昂贵, 此类设备主要在专 业领域供科学可视化使用.

另一类是轻便、可移动的穿戴式、手持式商用 设备, 如各类头戴式显示器和手机等. 随着视觉识 别和机器学习等技术的发展以及设备定位精度和 显示器分辨率的提升, 用户能以更加自然的方式 在沉浸式空间中交互, 同时降低由眩晕等引起的 负面影响. 随着沉浸式相关应用(如 VR 游戏、电影) 的发展，这类设备的使用率正逐渐提升.

\section{2 应用}

沉浸式可视化能够为几乎所有领域提供支持, 不仅有针对有形数据的探索与理解, 如科学可视 化 ${ }^{[57]}$ 和城市场景可视化 ${ }^{[16]}$, 也有针对抽象数据的 感知与分析, 如关系网络 ${ }^{[12]}$ 和散点图 ${ }^{[18]}$. 由于商 业沉浸式设备具有可移动性，因此，用户不再局限 于坐姿以及固定的物理场景，从而让分析者脱离 桌面办公环境而在数据采集的实地进行实时的分 析成为可能. 此外, 多人共同使用沉浸式设备为合 作式任务提供新的模式 ${ }^{[58]}$; 基于个人数据(如旅游 日志、社交媒体)的个人可视化需求也为沉浸式可 视化融人日常生活提供了契机 ${ }^{[35,59]}$. 总而言之, 随 着沉浸式技术的日益成熟以及研究者对广阔设计 空间的探索，人们将能够免除交互界面的限制，以 更自然且舒适的方式与数据进行高效的沟通, 在 众多应用中以沉浸的方式对数据进行探索、理解与 决策.

\section{3 展 望}

沉浸式技术正被应用于更广泛的领域中，而 各个应用也对沉浸式的可视化设计、工作流程和使 用方式提出了问题与挑战, 许多课题仍待相关研 究者的进一步探索.

在可视化设计中，一项挑战是如何结合多通 道感知的设计促进数据的理解与分析. 现有的分 析系统一般仅在立体视觉感知方面设计数据的表 达, 如时空立方体 ${ }^{[21-22]}$ 以及三维空间中边与轨迹 
的设计 ${ }^{[12,23-24]}$, 用户感知数据的方式有限. 触觉 ${ }^{[14]}$ 、 嗅觉 ${ }^{[27]}$ 等感知的辅助能够解决视觉遮挡的不便、 提升对数据的观察能力, 但用户的观察范围仅限 于坐姿或站立, 并未充分地利用其所允许的所有 移动空间释放空间感知与记忆能力. 因此, 各种感 知的组合方式、适用的分析任务及其效率尚待探 索. 另一项挑战是如何设计新的工作流程, 以适用 于临场可视化, 让可视分析走出实验室而进人实 地分析场景. 传统分析流程脱离实地场景, 用户能 够按照可视化分析流程完成相应任务. 而在临场 可视化中, 还需要考虑实地分析本身的流程以及 环境的限制. 这对可视化系统的性能、流程等提出 了新的设计需求. Whitlock 等 ${ }^{[60]}$ 将手机等 AR 设备 运用于公共场所避险等实地研究场景中, 将数据 的可视分析置于真实的环境内，从而提升用户在 现场分析中收集、分享数据以及决策的效率. 然而, 对于何种任务适用于临场解决、如何处理非地理类 型临场可视分析等问题仍有待探索.

在可视化交互方面，由于目前设备固有限制， 如头戴式 AR 设备中手势识别的精确性较低会让 用户在长时间使用下产生瘦劳与不适等, 相关研 究旨在降低三维手柄精确操作的难度 ${ }^{[42]}$ 、使用有 形用户界面增加选择精度 ${ }^{[40]}$, 以及设计新颖手势 减轻疲劳 ${ }^{[44]}$. 然而, 这些交互方式往往仅限于某 一类数据(如轨迹数据或地图), 无法为其他数据类 型的分析提供指导框架. 此外, 随着远程及合作分 析模式的兴起，沉浸式界面如何协调多用户下人 们之间的交互，将用户的手势与凝视等丰富的交 互信息合理地表达给其他用户，从而简化交互操 作并降低工作负荷, 是当前研究的另一类趋势与 难点 ${ }^{[8]}$.

在沉浸式可视化设计工具方面，面对纷繁复 杂的可视化需求, 如满足普通用户的日常数据表 达需要 ${ }^{[35]}$ 以及可视化专业人士的数据分析需求 ${ }^{[54]}$, 可视化创作需要新颖的设计流程、工具包或系统. 目前二维可视化存在诸如 D3 等基于 Web 的工具 包，但其在沉浸式环境下的多通道感知的表达、交 互设计较为欠缺. 目前无论是基于桌面端还是终 端的可视化创作工具, 主要以构造可视化标记或 图表为主，并辅助刷选和链接等基本交互，缺乏其 余感知通道的设计与运用，以及对新颖的交互动 作设计或有形交互设备使用的支持. 因此，如何设 计出满足广阔的沉浸式可视化需求的工具包或系 统，是可视化创作的另一个难点.

\section{参考文献(References):}

[1] Lindow N, Baum D, Leborgne M, et al. Interactive visualization of RNA and DNA structures[J]. IEEE Transactions on Visualization and Computer Graphics, 2019, 25(1): 967-976

[2] Liu D Y, Weng D, Li Y H, et al. SmartAdP: visual analytics of large-scale taxi trajectories for selecting billboard locations[J]. IEEE Transactions on Visualization and Computer Graphics, 2017, 23(1): 1-10

[3] Nguyen D B, Zhang L, Laramee R S, et al. Unsteady flow visualization via physics based pathline exploration[C] //Proceedings of the IEEE Visualization Conference. Los Alamitos: IEEE Computer Society Press, 2019: 286-290

[4] Ye S N, Chen Z T, Chu X T, et al. ShuttleSpace: exploring and analyzing movement trajectory in immersive visualization[J]. IEEE Transactions on Visualization and Computer Graphics, 2021, 27(2): 860-869

[5] Munzner T. Visualization analysis and design[M]. Boca Rotan: CRC Press, 2014

[6] Keim D, Qu H M, Ma K L. Big-data visualization[J]. IEEE Computer Graphics and Applications, 2013, 33(4): 20-21

[7] Cook K A, Thomas J J. Illuminating the path: the research and development agenda for visual analytics[R]. Washington: Pacific Northwest National Lab, 2005

[8] Dwyer T, Marriott K, Isenberg T, et al. Immersive Analytics: an introduction[M] //Immersive analytics. Heidelberg: Springer, 2018: 1-23

[9] Kilteni K, Groten R, Slater M. The sense of embodiment in virtual reality[J]. Presence: Teleoperators and Virtual Environments, 2013, 22(1): 373-387

[10] Slater M, Wilbur S. A framework for immersive virtual environments (FIVE): speculations on the role of presence in virtual environments[J]. Presence: Teleoperators \& Virtual Environments, 1997, 6(6): 603-616

[11] Witmer B G, Singer M J. Measuring presence in virtual environments: a presence questionnaire[J]. Presence, 1998, 7(3): 225-240

[12] Kwon O H, Muelder C, Lee K, et al. A study of layout, rendering, and interaction methods for immersive graph visualization[J]. IEEE Transactions on Visualization and Computer Graphics, 2016, 22(7): 1802-1815

[13] Alper B, Hollerer T, Kuchera-Morin J A, et al. Stereoscopic highlighting: 2D graph visualization on stereo displays[J]. IEEE Transactions on Visualization and Computer Graphics, 2011, 17(12): 2325-2333

[14] Prouzeau A, Cordeil M, Robin C, et al. Scaptics and highlight-planes: immersive interaction techniques for finding occluded features in 3D scatterplots[C] //Proceedings of the CHI Conference on Human Factors in Computing Systems. New York: ACM Press, 2019: Article No.325

[15] Kwon O H, Muelder C, Lee K, et al. Spherical layout and rendering methods for immersive graph visualization[C] // Proceedings of the IEEE Pacific Visualization Symposium. Los Alamitos: IEEE Computer Society Press, 2015: 63-67

[16] Chen Z T, Wang Y F, Sun T C, et al. Exploring the design space of immersive urban analytics[J]. Visual Informatics, 2017, 1(2): $132-142$ 
[17] Renner R S, Velichkovsky B M, Helmert J R. The perception of egocentric distances in virtual environments-a review[J]. ACM Computing Surveys, 2013, 46(2): Article No.23

[18] Kraus M, Weiler N, Oelke D, et al. The impact of immersion on cluster identification tasks[J]. IEEE Transactions on Visualization and Computer Graphics, 2020, 26(1): 525-535

[19] Krekhov A, Krüger J. Deadeye: a novel preattentive visualization technique based on dichoptic presentation[J]. IEEE Transactions on Visualization and Computer Graphics, 2019, 25(1): 936-945

[20] Krekhov A, Cmentowski S, Waschk A, et al. Deadeye visualization revisited: investigation of preattentiveness and applicability in virtual environments[J]. IEEE Transactions on Visualization and Computer Graphics, 2020, 26(1): 547-557

[21] Ssin S Y, Walsh J A, Smith R T, et al. GeoGate: correlating geo-temporal datasets using an augmented reality space-time cube and tangible interactions[C] //Proceedings of the IEEE Conference on Virtual Reality and 3D User Interfaces. Los Alamitos: IEEE Computer Society Press, 2019: 210-219

[22] Wagner Filho J A, Stuerzlinger W, Nedel L. Evaluating an immersive space-time cube geovisualization for intuitive trajectory data exploration[J]. IEEE Transactions on Visualization and Computer Graphics, 2020, 26(1): 514-524

[23] Yang Y L, Dwyer T, Jenny B, et al. Origin-destination flow maps in immersive environments[J]. IEEE Transactions on Visualization and Computer Graphics, 2019, 25(1): 693-703

[24] Büschel W, Vogt S, Dachselt R. Augmented reality graph visualizations[J]. IEEE Computer Graphics and Applications, 2019, 39(3): 29-40

[25] Kraus M, Angerbauer K, Buchmüller J, et al. Assessing 2D and 3D heatmaps for comparative analysis: an empirical study[C] // Proceedings of the CHI Conference on Human Factors in Computing Systems. New York: ACM Press, 2020: 1-14

[26] Ivanov A, Danyluk K, Jacob C, et al. A walk among the data[J]. IEEE Computer Graphics and Applications, 2019, 39(3): 19-28

[27] Patnaik B, Batch A, Elmqvist N. Information olfactation: harnessing scent to convey data[J]. IEEE Transactions on Visualization and Computer Graphics, 2019, 25(1): 726-736

[28] Kong H K, Zhu W J, Liu Z C, et al. Understanding visual cues in visualizations accompanied by audio narrations[C] // Proceedings of the CHI Conference on Human Factors in Computing Systems. New York: ACM Press, 2019: Article No.50

[29] Siu A F, Sinclair M, Kovacs R, et al. Virtual reality without vision: a haptic and auditory white cane to navigate complex virtual worlds[C] //Proceedings of the CHI Conference on Human Factors in Computing Systems. New York: ACM Press, 2020 $1-13$

[30] Batch A, Cunningham A, Cordeil M, et al. There is no spoon: evaluating performance, space use, and presence with expert domain users in immersive analytics[J]. IEEE Transactions on Visualization and Computer Graphics, 2020, 26(1): 536-546

[31] Cordeil M, Cunningham A, Dwyer T, et al. ImAxes: immersive axes as embodied affordances for interactive multivariate data visualisation[C] //Proceedings of the 30th Annual ACM Symposium on User Interface Software and Technology. New York: ACM Press, 2017: 71-83

[32] Büschel W, Chen J, Dachselt R, et al. Interaction for immersive
analytics[M] //Immersive Analytics. Heidelberg: Springer, 2018: 95-138

[33] Chen Z T, Zeng W, Yang Z G, et al. LassoNet: deep lasso-selection of 3D point clouds[J]. IEEE Transactions on Visualization and Computer Graphics, 2020, 26(1): 195-204

[34] Leiva G, Nguyen C, Kazi R H, et al. Pronto: rapid augmented reality video prototyping using sketches and enaction[C] // Proceedings of the CHI Conference on Human Factors in Computing Systems. New York: ACM Press, 2020: 1-13

[35] Chen Z T, Su Y J, Wang Y F, et al. MARVisT: authoring glyph-based visualization in mobile augmented reality[J]. IEEE Transactions on Visualization and Computer Graphics, 2020, 26(8): 2645-2658

[36] Usher W, Klacansky P, Federer F, et al. A virtual reality visualization tool for neuron tracing[J]. IEEE Transactions on Visualization and Computer Graphics, 2018, 24(1): 994-1003

[37] Wacker P, Wagner A, Voelker S, et al. Heatmaps, shadows, bubbles, rays: comparing mid-air pen position visualizations in handheld AR[C] //Proceedings of the $\mathrm{CHI}$ Conference on $\mathrm{Hu}-$ man Factors in Computing Systems. New York: ACM Press, 2020: $1-11$

[38] Wagner Filho J A, Freitas C M D S, Nedel L. Comfortable immersive analytics with the VirtualDesk metaphor[J]. IEEE Computer Graphics and Applications, 2019, 39(3): 41-53

[39] Wagner Filho J A, Freitas C M D S, Nedel L. VirtualDesk: a comfortable and efficient immersive information visualization approach[J]. Computer Graphics Forum, 2018, 37(3): 415-426

[40] Cordeil M, Bach B, Cunningham A, et al. Embodied axes: tangible, actuated interaction for 3D augmented reality data spaces[C] //Proceedings of the CHI Conference on Human Factors in Computing Systems. New York: ACM Press, 2020: $1-12$

[41] Fikkert F W. Gesture interaction at a distance[D]. Enschede: University of Twente. Centre for Telematics and Information Technology, 2010

[42] Baloup M, Pietrzak T, Casiez G. RayCursor: a 3D pointing facilitation technique based on raycasting[C] //Proceedings of the CHI Conference on Human Factors in Computing Systems. New York: ACM Press, 2019: Article No.101

[43] Hurter C, Riche N H, Drucker S M, et al. FiberClay: sculpting three dimensional trajectories to reveal structural insights[J]. IEEE Transactions on Visualization and Computer Graphics, 2019, 25(1): 704-714

[44] Satriadi K A, Ens B, Cordeil M, et al. Augmented reality map navigation with freehand gestures[C] //Proceedings of the IEEE Conference on Virtual Reality and 3D User Interfaces. Los Alamitos: IEEE Computer Society Press, 2019: 593-603

[45] Yang Y L, Cordeil M, Beyer J, et al. Embodied navigation in immersive abstract data visualization: is overview+detail or zooming better for 3D scatterplots?[J]. IEEE Transactions on Visualization and Computer Graphics, 2021, 27(2): 1214-1224

[46] Yang Y L, Dwyer T, Marriott K, et al. Tilt Map: interactive transitions between choropleth map, prism map and bar chart in immersive environments[J/OL]. IEEE Transactions on Visualization and Computer Graphics, 2020, 14(8):1-12[2020-11-24]. https://doi.org/10.1109/tvcg.2020.3004137

[47] Sidenmark L, Clarke C, Zhang X S, et al. Outline pur- 
suits: gaze-assisted selection of occluded objects in virtual reality[C]//Proceedings of the $\mathrm{CHI}$ Conference on $\mathrm{Hu}$ man Factors in Computing Systems. New York: ACM Press, 2020: 1-13

[48] Kwok T C K, Kiefer P, Schinazi V R, et al. Gaze-guided narratives: adapting audio guide content to gaze in virtual and real environments[C] //Proceedings of the $\mathrm{CHI}$ Conference on $\mathrm{Hu}$ man Factors in Computing Systems. New York: ACM Press, 2019: Article No.491

[49] Alghofaili R, Sawahata Y, Huang H K, et al. Lost in style: gaze-driven adaptive aid for VR navigation[C] //Proceedings of the CHI Conference on Human Factors in Computing Systems. New York: ACM Press, 2019: Article No.348

[50] Abtahi P, Gonzalez-Franco M, Ofek E, et al. I'm a giant: walking in large virtual environments at high speed gains[C] //Proceedings of the CHI Conference on Human Factors in Computing Systems. New York: ACM Press, 2019: Article No.522

[51] Funk M, Müller F, Fendrich M, et al. Assessing the accuracy of point \& teleport locomotion with orientation indication for virtual reality using curved trajectories[C] //Proceedings of the CHI Conference on Human Factors in Computing Systems. New York: ACM Press, 2019: Article No.147

[52] Drogemuller A, Cunningham A, Walsh J, et al. Evaluating navigation techniques for 3D graph visualizations in virtual reality[C] //Proceedings of the International Symposium on Big Data Visual and Immersive Analytics. Los Alamitos: IEEE Computer Society Press, 2018: 1-10

[53] Sicat R, Li J B, Choi J Y, et al. DXR: a toolkit for building immersive data visualizations[J]. IEEE Transactions on Visualization and Computer Graphics, 2019, 25(1): 715-725

[54] Cordeil M, Cunningham A, Bach B, et al. IATK: an immersive analytics toolkit[C] //Proceedings of the IEEE Conference on Virtual Reality and 3D User Interfaces. Los Alamitos: IEEE Computer Society Press, 2019: 200-209

[55] Prouzeau A, Wang Y C, Ens B, et al. Corsican twin: authoring in situ augmented reality visualisations in virtual reality[C] // Proceedings of the International Conference on Advanced Visual Interfaces. New York: ACM Press, 2020: Article No.11

[56] Febretti A, Nishimoto A, Thigpen T, et al. CAVE2: a hybrid reality environment for immersive simulation and information analysis[C] //Proceedings of SPIE. Bellingham: Society of Photo-Optical Instrumentation Engineers, 2013, 8649: Article No.864903

[57] Verbraeck A, Eisemann E. Interactive black-hole visualization[J]. IEEE Transactions on Visualization and Computer Graphics, 2021, 27(2): 796-805

[58] Lee B, Hu X Y, Cordeil M, et al. Shared surfaces and spaces: collaborative data visualisation in a co-located immersive environment[J]. IEEE Transactions on Visualization and Computer Graphics, 2021, 27(2): 1171-1181

[59] Huang D D, Tory M, Aseniero B A, et al. Personal visualization and personal visual analytics[J]. IEEE Transactions on Visualization and Computer Graphics, 2015, 21(3): 420-433

[60] Whitlock M, Wu K K, Szafir D A. Designing for mobile and immersive visual analytics in the field[J]. IEEE Transactions on Visualization and Computer Graphics, 2020, 26(1): 503-513 